\title{
Giant hole and circular superflow in a fast rotating Bose-Einstein condensate
}

\author{
Kenichi Kasamatsu ${ }^{1}$, Makoto Tsubota ${ }^{1}$, and Masahito Ueda $^{2}$ \\ ${ }^{1}$ Department of Physics, Osaka City University, Sumiyoshi-Ku, Osaka 558-8585, Japan \\ ${ }^{2}$ Department of Physics, Tokyo Institute of Technology, Meguro-ku, Tokyo 152-8551, Japan
}

(Dated: October 28, 2018)

\begin{abstract}
A fast rotating Bose-Einstein condensate confined in a quadratic-plus-quartic potential is found to dynamically generate a "giant vortex" that absorbs all phase singularities into a central low density hole, thereby sustaining a quasi-one-dimensional circular superflow at a supersonic speed.
\end{abstract}

PACS numbers: 03.75.Fi, 67.40.Db

Gaseous Bose-Einstein condensates (BECs) are an extremely versatile testing ground for superfluidity particularly when an externally driven rotation exists. Through a number of experimental [1, 2, 3, 4] and theoretical studies [5] on rotating BECs, the argument now extends to the fast rotating regime [6, 6, 8, 9, 10]. For a harmonic trapping potential $(1 / 2) m \omega_{\perp}^{2} r^{2}$, the centrifugal force prevents a BEC from rotating at frequency $\Omega$ beyond the radial trapping frequency $\omega_{\perp}$. However, a recent experimental development [11] should enable one to create a confinement potential tighter than harmonic, thus opening a possible method to explore the nature of fast rotating BECs.

In this article, we extend our previous studies [12] to the dynamics of vortex lattice formation of a $\mathrm{BEC}$ in a quadratic-plus-quartic potential with $\Omega$ greater than $\omega_{\perp}$. Here, the stable vortex configuration, realized at a value of $\Omega$ much greater than $\omega_{\perp}$, is not triangular but rather a circular array around a central low density hole [see Fig. 1(e) below]. This hole is created where singly quantized vortices are close together but do not completely overlap. Surprisingly, as $\Omega$ is further increased, all the vortices are absorbed into the central low density hole, around which persistent current circulates. This superflow is supersonic, which offers an avenue of research in superfluidity, because it has been difficult to obtain a circular superflow in superfluid helium [13]. For an atomic-gas BEC, a circular superflow can occur in an ideal situation and the superfluid speed is easily controlled by changing $\Omega$. This opens up possibilities of studying phase slippage 14 and the stability of a controlled superflow.

We consider a two-dimensional system subject to rotation $\boldsymbol{\Omega}=\Omega \hat{\mathbf{z}}$ by assuming translation symmetry along the $z$ axis. In a frame rotating with frequency $\Omega$ around the $z$ axis, the dynamics of a condensate "wave function" $\Psi$ is described by the Gross-Pitaevskii equation

$$
i \frac{\partial \Psi}{\partial t}=\left(-\nabla^{2}+V_{\text {trap }}-\mu+C|\Psi|^{2}-\Omega \hat{L}_{z}\right) \Psi
$$

where $\mu$ is the chemical potential and the wave function is normalized to unity: $\int d x d y|\Psi|^{2}=1$. The units of energy, length, and time are given by the corresponding scales of the harmonic potential, that is, $\hbar \omega_{\perp}, b_{\perp}=$ $\sqrt{\hbar / 2 m \omega_{\perp}}$, and $\omega_{\perp}^{-1}$, respectively, where $m$ is the atomic mass and $\omega_{\perp}$ the frequency of the harmonic potential.
The strength of the mean-field interaction between atoms is characterized by $C$ which is proportional to the $s$ wave scattering length $a_{s}$ as $C=8 \pi N_{2 \mathrm{D}} a_{s}$, where $N_{2 \mathrm{D}}$ is the particle number per unit length along the $z$ axis. The trapping potential is $V_{\text {trap }}(r)=r^{2} / 4+k r^{4} / 16$, where $r^{2} \equiv x^{2}+y^{2}$ and $k$ is the strength of the quartic term. The quartic part of $V_{\text {trap }}$ allows us to increase $\Omega$ above $\omega_{\perp}$. For a rotating condensate an effective potential in the radial direction is

$$
V_{\text {trap }}^{\mathrm{eff}}(r)=\frac{1}{4}\left[\left(1-\Omega^{2}\right) r^{2}+\frac{1}{4} k r^{4}\right]
$$

(a)

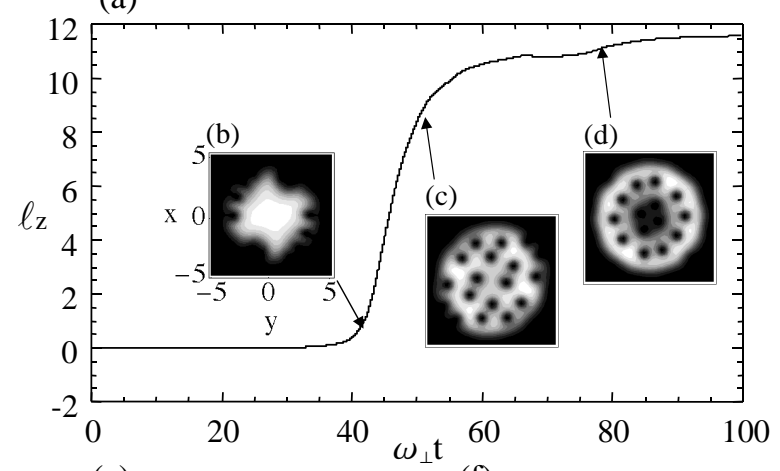

(e)

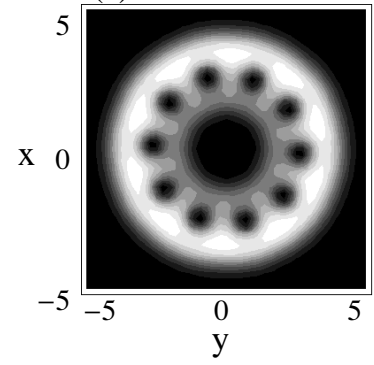

(f)

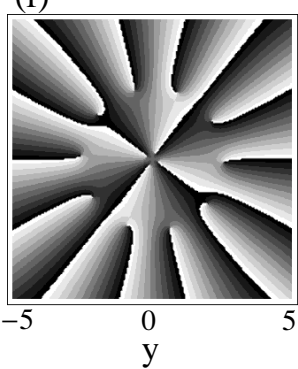

FIG. 1: Time development of the angular momentum per atom $\ell_{z}$ (a) and the condensate density $|\Psi|^{2}$ for $\Omega=2.5$ (b)(e). Times $\omega_{\perp} t=42$ (b), 50 (c), and 78 (d). The lower panel shows the density (e) and phase (f) profiles of the final stationary state of this dynamic system. The value of the phase varies continuously from 0 (black) to $2 \pi$ (white). The discontinuity lines between black and white are the branch cuts in the complex plane. The termination of each branch cut is a vortex, or equivalently a phase defects. 
This potential has a "Mexican hat" structure for $\Omega>1$, which changes the dynamics of vortex lattice formation as shown below.

Now, we investigate the dynamics of vortex lattice formation [15]. The stationary solution of Eq. (11) for a nonrotating trap is used as the initial state. The rotating drive is suddenly turned on by introducing a small anisotropy of the harmonic trap as $\left(\epsilon_{x} x^{2}+\epsilon_{y} y^{2}\right) / 4$ [1, 12 . Because the transition from a nonvortex state to a vortex state requires energy dissipation, a phenomenological damping constant $\gamma$ is put in Eq. (11) by replacing $i \partial / \partial t$ with $(i-\gamma) \partial / \partial t$ [12, 16]. Finally, the small trap anisotropy $\epsilon_{x, y}$ is turned off adiabatically to obtain the axisymmetric stationary state.

Numerical simulations were done for several values of $\Omega$ at fixed $C=250$ and $k=1$. Dynamics that are unique to the quadratic-plus-quartic potential occur when $\Omega>1$. Figure 11 shows the time development of the angular momentum per atom $\ell_{z}=\int d x d y \Psi^{*} \hat{L}_{z} \Psi$, and the density and phase profiles for $\Omega=2.5$. The ripples are excited on the surface [Fig. 1(b)], and some ripples penetrate into the condensate and develop into vortex cores [Fig. 1(c)]. Some penetrating vortices move toward the center, merging together to make a hole at the center [Fig. 11(d)]. During the period between that shown in Fig. 1(c) and that in Fig. 11(d), additional vortices entered the condensate successively, which gradually increased the angular momentum. The density and phase profiles of the final steady state are shown in Figs. 11(e) and 1 $1(\mathrm{f})$, respectively. A hole appears at the center, around which some vortices form a circular array. Furthermore, near the center of the condensate, some phase defects come very close to each other without overlapping [Fig. 11(f)].

As $\Omega$ is further increased, all vortices generated from the condensate surface are absorbed into the central low density hole as shown in Fig. 2(a). Note that this central hole is composed of 24 singly quantized vortices packed together [Fig. 2(b)]. This packing is possible for high rotation frequencies $(\Omega>1)$ because the centrifugal force decreases the condensate density near the center; thus packing vortices costs little energy. The minimum of Eq. (2) determines the radius of the ring condensate in Fig. 2 as $R=\sqrt{2\left(\Omega^{2}-1\right) / k}$. Here we call a set of vortices such as that in Fig. 2 a "giant vortex" to indicate that a number of phase defects are contained in a single hole [see Fig. 2(b)]. Such a density depression with a concentration of vorticity occurs in rotating superfluid helium [17.

Figures 1 (f) and 2(b) show that the phase singularities do not completely overlap. The condensate inside the hole has a very low density and hence a long coherence length, so that the vortex cores have a nearly uniform density. Thus, only the locations of singularities matter and one can assume that the interaction energy between the singularity points is proportional to $-\rho \kappa^{2} \ln d$, where $\rho$ is the condensate density, $\kappa$ the quantum circulation, and $d$ the distance between defects. The logarithmic divergence at $d \rightarrow 0$ prevents the phase singularities from (a)

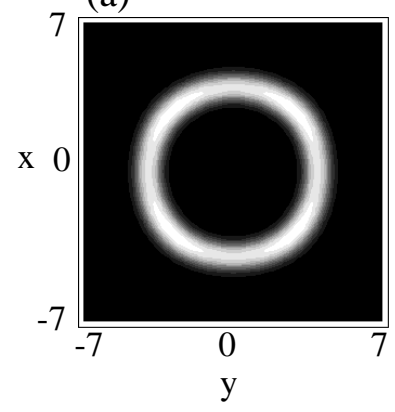

(b)

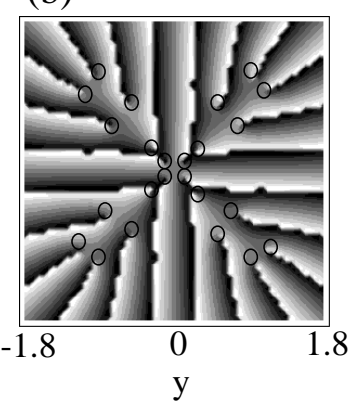

FIG. 2: The condensate density (a) and phase field (b) of the vortex state with circulation $24 h / m$ for $\Omega=3.2$. The phase field region in (b) is the central low density region of (a), where 24 phase defects are marked by open circles.

merging together.

The appearance of such a density hole is relevant to recent studies on fast rotating BECs that used the ThomasFermi and Wigner-Seitz approximation [6, 8]. Fischer and Baym evaluated the vortex core size self-consistently by minimizing the Gross-Pitaevskii energy functional and using a variational wave function [8]; they showed that vortex cores never overlap, in agreement with our finding. They also showed that the giant vortex state is energetically favorable at large $\Omega$. Although the Thomas-Fermi approximation accurately describes stationary solutions such as that in Fig. 1 (e), it fails when the width of the ring condensate becomes thin such as that in Fig. 2. Even if $\Omega$ is not large enough, a pinning potential might be used to stabilize the giant vortex 18].

For sufficiently large $\Omega$, the ring structure of Fig. 2(a) offers some insights into circular superflow, which has been studied for a torus [13]. The superfluid velocity is given by the gradient of the phase of the wave function as $\mathbf{v}_{s}=(\hbar / m) \nabla \theta$; the circulation along a closed loop is quantized as $\oint \mathbf{v}_{s} \cdot d \mathbf{l}=\kappa n$ with $\kappa \equiv h / m$ being the quantum circulation. The quantum number $n$ is equal to the number of branch cuts of the phase field along the loop or, equivalently, the number of phase defects in the low density hole. If there is sufficient dissipation, some vortices in the low density hole should spiral out of the condensate when $\Omega$ is abruptly decreased from that of Fig. 2(a). The motion of these vortices across the circular superflow causes $v_{s}$ to decay. This is known as phase slippage [14], which is a characteristic feature of superfluidity.

Measurement of the angular momentum of the giant vortex allows the phase slippage to be observed well under control. For example, phase slippage was observed in helium superflow through microapertures [19], but it was difficult to control single phase slips and giant slips. Figure 3 shows the time development of the angular momentum and phase field resulting from a decrease in $\Omega$ from $\Omega=3.5(n=32)$ to $\Omega=3.2$. Here we assumed a small anisotropy in the trapping frequencies to break the 
rotational symmetry. Some phase defects are released from the low density hole, jostling at the inner border of the ring condensate. We see that four vortices cross the superflow by phase slippage and then escape outside (only one escape is shown in Fig. 3), thus decreasing the angular momentum.

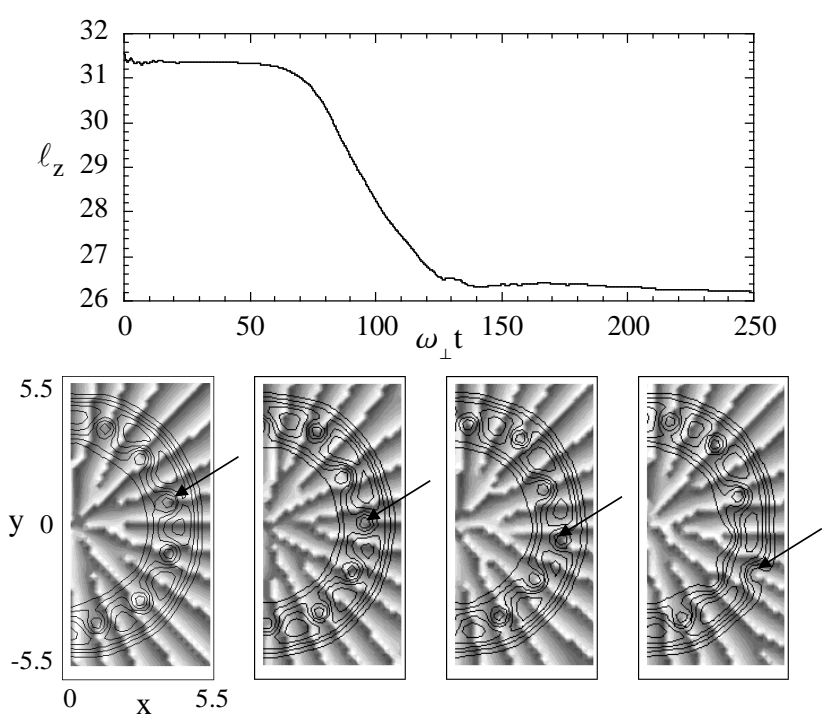

FIG. 3: The top figure shows the time evolution of the angular momentum per atom $\ell_{z}$ for a change of $\Omega$ from 3.5 to 3.2. The bottom figures show the phase profiles and line contours of the density for half of the $x-y$ plane at $\omega_{\perp} t=102,107,112$, and 116 from left to right. Arrows show how one vortex escapes via phase slippage.

When the ring radius $R$ is sufficiently large, the circular flow becomes quasi-one-dimensional for the following reason. The radial wave function of the stationary giant vortex state is obtained by substituting $\Psi \cong f(r) e^{i n \theta}$ into Eq. (1):

$$
\left[-\frac{\partial^{2}}{\partial r^{2}}-\frac{1}{r} \frac{\partial}{\partial r}+\frac{n^{2}}{r^{2}}+V_{\text {trap }}+C|f|^{2}-n \Omega\right] f=\mu f .
$$

The excitation of the radial component is easily obtained by writing $f+\delta f$; the effective potential for $\delta f$ is then given by

$$
V_{\text {eff }}(r)=V_{\text {trap }}+\frac{n^{2}}{r^{2}}+2 C|f(r)|^{2}-\mu-n \Omega
$$

Because $n=\Omega R^{2} / 2$ in the limit of a rigid body rotation [6, 7], the radial zero-point energy around the minimum of the potential $V_{\text {trap }}+n^{2} / r^{2}$ becomes larger than the mean-field interaction strength $C|f(r)|^{2}$ for large $R$. Such a high excitation gap freezes the motion of the radial wave function while keeping the motion free along the $\theta$ direction. To investigate the stability of this one-dimensional superflow, we substitute $\Psi(r, \theta, t)=f(r) \psi(\theta, t)$ into Eq. (11) to obtain

$$
i \frac{\partial \psi}{\partial t}=\left(-\frac{1}{R^{2}} \frac{\partial^{2}}{\partial \theta^{2}}+E_{r}+C P|\psi|^{2}-i \Omega \frac{\partial}{\partial \theta}\right) \psi,
$$

where

$$
\begin{gathered}
E_{r}=\frac{1}{A} \int r d r f^{*}\left[-\frac{\partial^{2}}{\partial r^{2}}-\frac{1}{r} \frac{\partial}{\partial r}+V_{\text {trap }}-\mu\right] f, \\
R^{-2} \simeq \frac{1}{A} \int r d r|f|^{2} r^{-2}, \quad P=\frac{1}{A} \int r d r|f|^{4}
\end{gathered}
$$

with $A=\int r d r|f|^{2}$. The wave function is linearized as $\psi(\theta, t)=e^{-i \mu t}\left\{e^{i n \theta}+\eta\left[e^{-i \omega t} u(\theta)+e^{i \omega^{*} t} v^{*}(\theta)\right]\right\}$. The resulting eigenvalue problem with the ansatz $u(\theta)=$ $u_{q} e^{i(n+q) \theta}$ and $v(\theta)=v_{q} e^{i(-n+q) \theta}$ yields the dispersion relation

$$
\omega_{q, \pm}=\frac{q}{R}\left\{\left(\frac{2 n}{R}-R \Omega\right) \pm \sqrt{\left(\frac{q}{R}\right)^{2}+2 C P}\right\},
$$

where $q / R$ is the wave number of the excitation. The second term on the right-hand side of Eq. (6) gives the sound velocity $c=\sqrt{2 C P}$. The superflow is stable when $v_{s}<c$ according to the Landau criterion. The values of the parameters and the form of $f(r)$ of Fig. 2(a) yield $c=3.75$ and the circular flow velocity $v_{s}=2 n / R \simeq 12$ (in units of $b_{\perp} \omega_{\perp}$ ). Because $v_{s}>c$, the circular flow is supersonic and energetically unstable. (a)

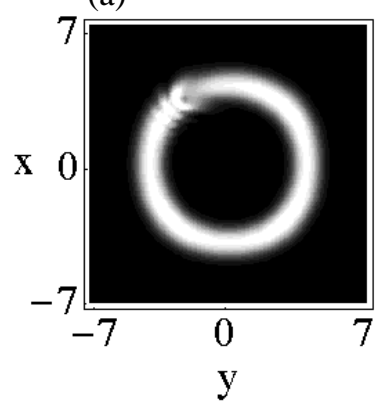

(b)

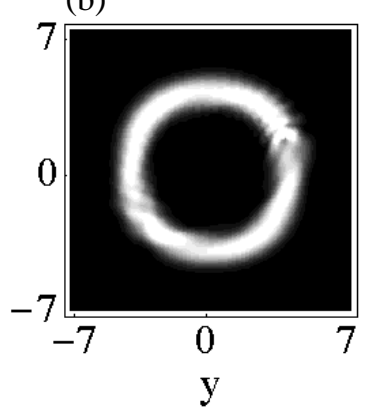

FIG. 4: Snapshots of the numerical simulation of a giant vortex state (Fig. 2) that is perturbed by an impurity potential. Parameter values for $V_{\mathrm{imp}}$ are $V_{0}=100, \sigma=0.1, x_{0}=3.94$ and $y_{0}=0$. The impurity potential is at rest in the laboratory frame, and thus rotates in the rotating frame.

Impurities and walls can cause dissipation for a supersonic superflow. For example, Ref. [20] used the Gross-Pitaevskii equation for bulk condensates and ordinary trapped condensates to show that the dissipation of the superflow is caused by vortex-pair creation at an impurity object. This was shown experimentally [21. However, this does not apply to the present case of quasi-one-dimensional superflow because of the tight radial confinement [22]. To show the influence of impurities, we introduce an impurity potential of Gaussian form as $V_{\text {imp }}=V_{0} \exp \left[-\left\{\left(x-x_{0}\right)^{2}+\left(y-y_{0}\right)^{2}\right\} / \sigma^{2}\right]$ (at rest in the laboratory frame), where $\left\{x_{0}, y_{0}\right\}$ is the impurity position. Figure 1 shows an example of the destabilization process of the giant vortex state by $V_{\mathrm{imp}}$, for which $V_{0}$ is chosen to be larger than $V_{\text {trap }}(R)$ and $\sigma$ smaller than 
the width of the ring condensate. Because $v_{s}$ exceeds the Landau critical velocity $c$, the impurity excites the longitudinal density waves along the ring condensate, thus producing a drag force. Due to the presence of dissipation and the rotating drive, the system reaches a steady state as shown in Fig. 14(b). Therefore, the quadratic-plusquartic system offers a scheme for studying the stability of a quasi-one-dimensional superflow.

In conclusion, a fast rotating BEC in a quadratic-plusquartic potential can generate a giant vortex and circular superflow around it by absorbing singly quantized vortices into a central low density hole. The circular superflow is quasi-one-dimensional and goes at a supersonic speed. We thus hope that this system will encourage experiments to study low dimensional superfluidity in atomic-gas BECs.

M.T. acknowledges support by a Grant-in-Aid for Scientific Research (Grant No. 12640357) by the Japan Society for the Promotion of Science. M.U. acknowledges support by a Grant-in-Aid for Scientific Research (Grant No. 11216204) by the Ministry of Education, Science, Sports, and Culture of Japan, and by the Toray Science Foundation.
[1] K. W. Madison, F. Chevy, W. Wohlleben, and J. Dalibard, Phys. Rev. Lett 84, 806 (2000).

[2] J. R. Abo-Shaeer, C. Raman, J. M. Vogels, and W. Ketterle, Science 292, 476 (2001).

[3] P. C. Haljan, I. Coddington, P. Engels, and E. A. Cornell, Phys. Rev. Lett. 87, 210403 (2001).

[4] E. Hodby, G. Hechenblaikner, S. A. Hopkins, O. M. Maragó, and C. J. Foot, Phys. Rev. Lett. 88, 010405 (2002).

[5] For a review, see A. L. Fetter, and A. A. Svidzinsky, J. Phys.: Condens. Matter 13, R135 (2001).

[6] A. L. Fetter, Phys. Rev. A 64, 063608 (2001).

[7] D. L. Feder and C. W. Clark, Phys. Rev. Lett. 87, 190401 (2001).

[8] U. R. Fischer and G. Baym, cond-mat/0111443.

[9] E. Lundh, Phys. Rev. A 65, 043604 (2002).

[10] T. L. Ho, Phys. Rev. Lett. 87, 060403 (2001); N. R. Cooper, N. K. Wilkin, and J. M. F. Gunn, ibid. 87, 120405 (2001); S. Viefers, T. H. Hansson, and S. M. Reimann, Phys. Rev. A 62, 053604 (2000).

[11] T. Kuga, Y. Torii, N. Shiokawa, T. Hirano, Y. Shimizu, and H. Sasada, Phys. Rev. Lett. 78, 4713 (1997).

[12] M. Tsubota, K. Kasamatsu, and M. Ueda, Phys. Rev. A 65, 023603 (2002).

[13] R. J. Donnely, Quantized Vortices in Helium II (Cambridge University Press, Cambridge, 1991).

[14] P. W. Anderson, Rev. Mod. Phys. 38, 298 (1966).

[15] The numerical simulations were done with an alternatingdirection implicit method in the $256 \times 256$ discretized space with the spatial region $[-7,7] \times[-7,7]$.

[16] In this article, we assume $\gamma=0.03$. This value was obtained by S. Choi, S. A. Morgan, and K. Burnett, Phys. Rev. A 57, 4057 (1998) by fitting a numerical simulation of the dissipative Gross-Pitaevskii equation with the experimental data on collective excitation by M.-O. Mewes et al. Phys. Rev. Lett. 77, 988 (1996). Collisions between condensate and noncondensate atoms might cause dissipation. A detailed analysis of this will be reported elsewhere.

[17] P. L. Marston and W. M. Fairbank, Phys. Rev. Lett. 39, 1208 (1977).

[18] T. P. Simula, S. M. M. Virtanen, and M. M. Salomaa, Phys. Rev. A 65, 033614 (2002).

[19] E. Varoquaux, O. Avenel, Y. Mukharsky, and P. Hakonen, Quantized Vortex Dynamics and Superfluid Turbulence," edited by C. F. Barenghi, R. J. Donnelly, and W. F. Vinen (Springer, Berlin, 2001), and references therein.

[20] T. Frisch, Y. Pomeau, and S. Rica, Phys. Rev. Lett. 69, 1644 (1992); B. Jackson, J. F. McCann, and C. S. Adams, ibid. 80, 3903 (1998).

[21] C. Raman et al. Phys. Rev. Lett. 83, 2502 (1999); R. Onofrio et al., ibid. 85, 2228 (2000).

[22] Recently we learned of a paper [C. K. Law, C. M. Chan, P. T. Leung, and M. -C. Chu, Phys. Rev. Lett. 85, 1598 (2000)] that shows that the one-dimensional, supersonic superflow is stable for a particular form of the impurity potential. 